Supporting Information :

\title{
Sensing of Hydrogen Sulfide Gas in Raman-silent Region based on Gold Nano-bipyramids (Au NBPs) encapsulated by Zeolitic Imidazolate Frameworks-8
}

Jiaming Chen ${ }^{a, b}$, Longhua Guo ${ }^{c *}$, Lifen Chen ${ }^{c}$, Bin Qiu ${ }^{b}$, Guolin Hong ${ }^{a *}$, and Zhenyu Lin ${ }^{b *}$

a Department of Laboratory Medicine, The First Affiliated Hospital of Xiamen University, Xiamen Key Laboratory of Genetic Testing; Xiamen, Fujian 361005, China;

${ }^{\mathrm{b}}$ College of Chemistry, Fuzhou University, MOE Key Laboratory for Analytical Science of Food Safety and Biology, Fujian Provincial Key Laboratory of Analysis and Detection Technology for Food Safety, Fuzhou, Fujian, 350116, China;

${ }^{\mathrm{c}}$ College of Biological, Chemical Sciences and Engineering, Jiaxing University, Jiaxing, Zhejiang, 314001, China.

Correspondence and requests for materials should be addressed to E-mail: guolh@zjxu.edu.cn (Longhua Guo); E-mail: 18860089899@139.com (Guolin Hong); E-mail: zylin@fzu.edu.cn (Zhenyu Lin).

\section{Table of Content:}

Figure S1 The TEM image of Au NBPs@PVP

Figure S2 The powder X-Ray Diffraction (XRD) patterns

Figure S3 The Raman spectra of Au NBPs@ZIF-8 exposed to the different environment

Figure S4 The Raman spectra of Au NBPs@ZIF-8 at different storage time

Figure S5 The Raman spectra of Au NBPs@ZIF-8 with three different fish meat

Figure S6 Standard colorimetric card of $\mathrm{H}_{2} \mathrm{~S}$ content assay kit

Table S1 Comparison of the performance of different sensors for $\mathrm{H}_{2} \mathrm{~S}$ detection 


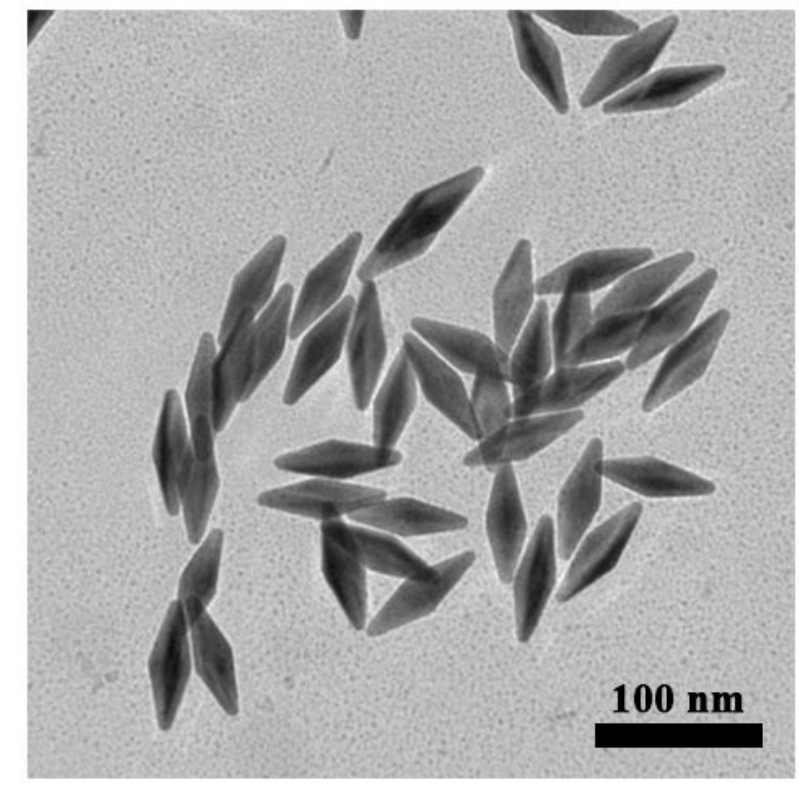

Figure S1 The TEM image of PVP stabilized Au NBPs.

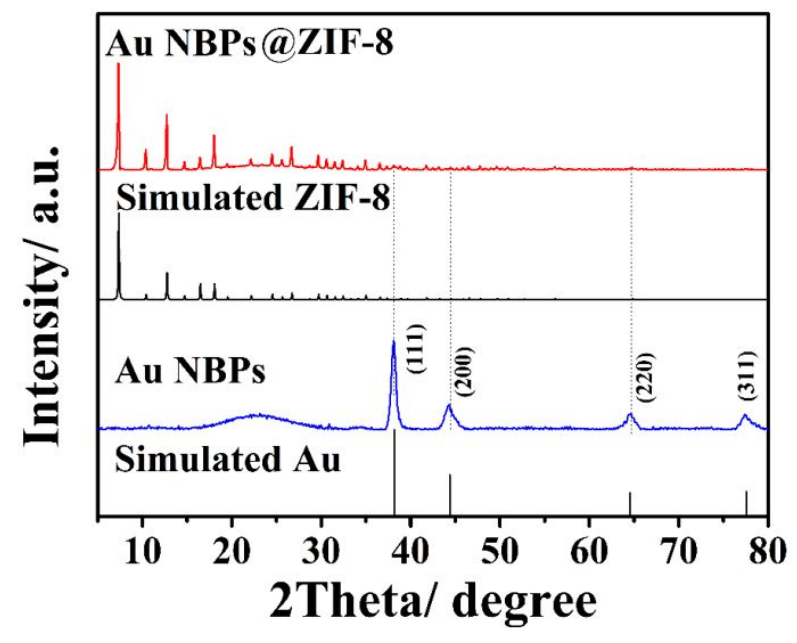

Figure S2 Powder X-Ray Diffraction (XRD) patterns simulated for Au NBPs and ZIF8 and experimentally determined for as-synthesized Au NBPs@ZIF-8.Au NBPs@ZIF8 yielded diffractograms containing peaks are corresponding to both ZIF-8 $\left(2 \theta=5.0 \sim 40^{\circ}\right)$ and $\mathrm{Au}$ NBPs $\left(2 \theta=38.2^{\circ}, 44.4^{\circ}, 64.6^{\circ}\right)$ 


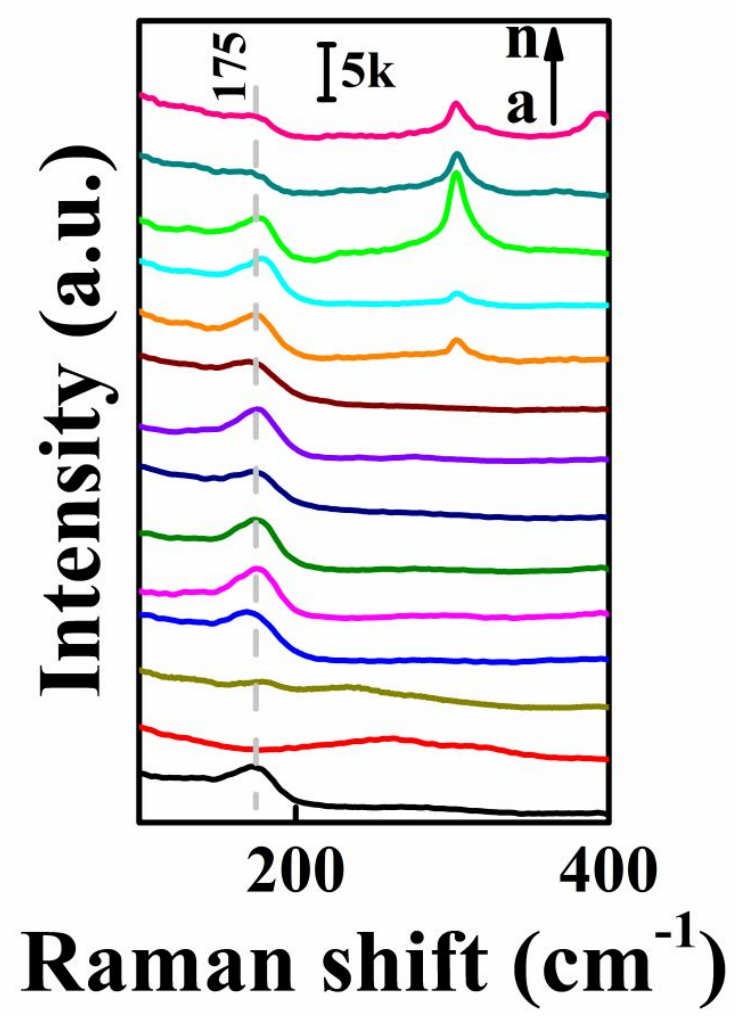

Figure S3 The Raman spectra of a-n represents Au NBPs@ZIF-8 in presence of air, $\mathrm{H}_{2} \mathrm{~S}(20 \mu \mathrm{M})$, and $500 \mu \mathrm{M}$ of $\mathrm{HCl}$, ethanol, toluene, $\mathrm{NH}_{3}, \mathrm{CH}_{4}, \mathrm{CO}_{2}, \mathrm{SO}_{2}$, dimethyl disulfide, L-Cysteine, glutathione, mercaptoacetic acid, and 4-Aminobenzenethiol, respectively, $10 \mathrm{~min}$.
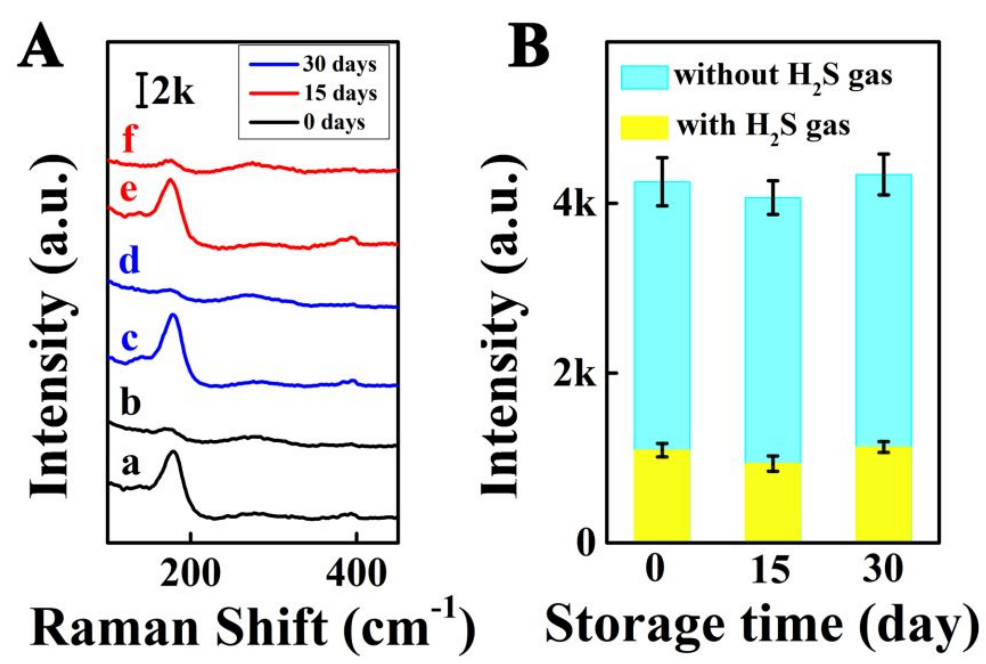

Figure S4 (A) The SERS spectra were collected from the Au NBPs@ZIF-8 substrates after 0 days, 15 days, and 30 days. (a), (c), and (e) represent the SERS spectra of the substrates before exposing to $\mathrm{H}_{2} \mathrm{~S}$ gas; (b), (d), and (f) represent the SERS spectra of the substrates after exposing to $\mathrm{H}_{2} \mathrm{~S}$ gas. The $\mathrm{H}_{2} \mathrm{~S}$ gas was $2.0 \mathrm{mM}$. (B) The variation of intensities at $175 \mathrm{~cm}^{-1}$ (mean $\pm \mathrm{SD}$ ) before and after exposure to $\mathrm{H}_{2} \mathrm{~S}$ gas for different storage time. The wavelength of laser: $785 \mathrm{~nm}$. 


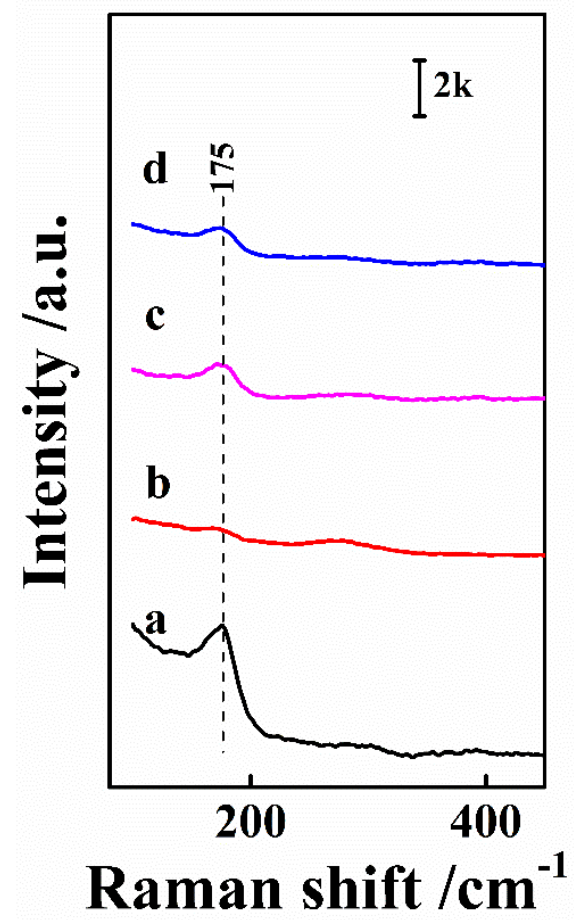

Figure S5 The Raman spectra of Au NBPs@ZIF-8 with three different fish meat: (a) Au NBPs@ZIF-8, (b) Cololabis saira, (c) Hypomesus olidus, (d) Larimichthys polyactis, for five minutes. All of the fish meat have been stored for $48 \mathrm{~h}$, at $25^{\circ} \mathrm{C}$.

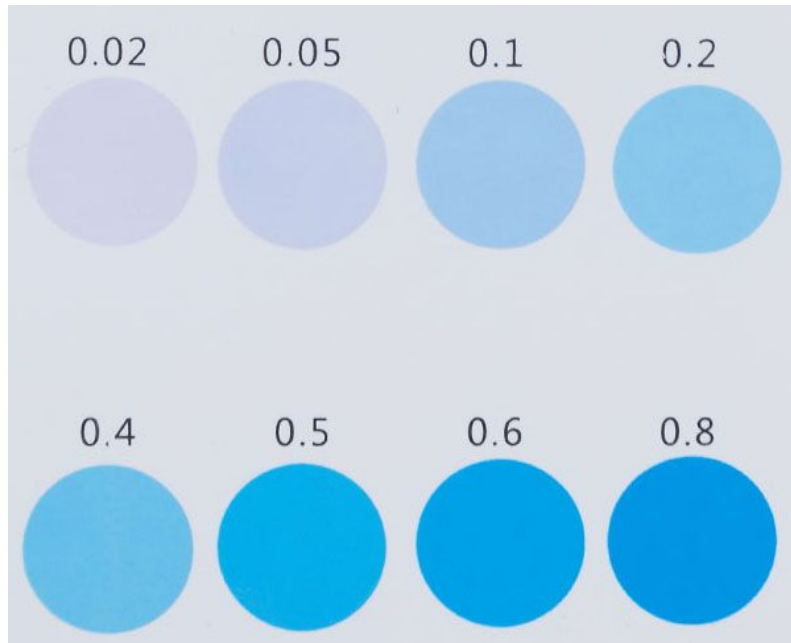

Figure S6 Standard colorimetric card of $\mathrm{H}_{2} \mathrm{~S}$ content assay kit (mg/ L).

Table S1. Comparison of the performance of different sensors for $\mathrm{H}_{2} \mathrm{~S}$ detection

$\begin{array}{llll}\text { Sensors } & \text { Detection time } & \text { Detection Range }\end{array}$




$\begin{array}{llll}\mathrm{Au} N \mathrm{NP} @ \mathrm{CuO} & 10 \mathrm{~min} & 0.1 \mathrm{ppb}-1 \mathrm{ppm} & 0.1 \mathrm{ppb}\end{array}$

$\begin{array}{llll}\mathrm{WS}_{2} \text { sensor } & 1-6 \mathrm{~min} & \text { N.A. } & 20 \mathrm{ppb}\end{array}$

$\mathrm{MoSe}_{2}$ sensor

N.A.

$50 \mathrm{ppb}-5.45 \mathrm{ppm}$

$50 \mathrm{ppb}$

3
$\mathrm{CuO} / \mathrm{MoS}_{2}$ sensor

N.A.

1 ppb - 100 ppm

$1 \mathrm{ppb}$

4

Colorimetric sensor of Au NP seconds $0.1-1.5$ ppm $0.5 \mathrm{ppm}$ 5 aggregation
Au NBPs@ZIF-8
SERS sensor ${ }^{\mathrm{a}}$
$5 \min$
0.13 ppb - 1300 ppm
$0.11 \mathrm{ppb}$
This work

a The concentration converted from molar concentration to parts per billion (ppb, vol) and parts per million (ppm, vol) mass to volume in the air.

\section{Reference}

(1) Bao, H.; Zhang, H.; Fu, H.; Zhou, L.; Zhang, P.; Li, Y.; Cai, W., Ultrathin layer solid transformation-enabled-surface enhanced Raman spectroscopy for trace harmful small gaseous molecule detection. Nanoscale Horizons 2020, 5, 739-746. 
(2) Asres, G. A.; Baldoví, J. J.; Dombovari, A.; Järvinen, T.; Lorite, G. S.; Mohl, M.; Shchukarev, A.; Paz, A. P.; Xian, L.; Mikkola, J.-P., Ultrasensitive H 2 S gas sensors based on p-type WS 2 hybrid materials. Nano Research 2018, 11, 4215-4224.

(3) Jha, R. K.; D'Costa, J. V.; Sakhuja, N.; Bhat, N., MoSe2 nanoflakes based chemiresistive sensors for ppb-level hydrogen sulfide gas detection. Sensors Actuators B: Chem. 2019, 297, 126687

(4) Zhang, D.; Wu, J.; Cao, Y., Ultrasensitive H2S gas detection at room temperature based on copper oxide/molybdenum disulfide nanocomposite with synergistic effect. Sensors Actuators B: Chem. 2019, 287, 346-355.

(5) Zhang, Z.; Chen, Z.; Wang, S.; Qu, C.; Chen, L., On-site visual detection of hydrogen sulfide in air based on enhancing the stability of gold nanoparticles. ACS applied materials \& interfaces 2014, 6, 6300-6307. 